\title{
THE CORSAIRS OF SAINT-MALO
}<smiles>[As]</smiles> 


\section{THE MIDDLE RANGE}

\section{Edited by Peter S. Bearman and Shamus R. Khan}

The Middle Range, coined and represented by Columbia sociologist Robert Merton, is a style of work that treats theory and observation as a single endeavor. This approach has yielded the most significant advances in the social sciences over the last half century; it is a defining feature of Columbia's department. This book series seeks to capitalize on the impact of approaches of the middle range and to solidify the association between Columbia University and its Press.

The Conversational Firm: Rethinking Bureaucracy in the Age of Social Media, Catherine J. Turco

Working for Respect: Community and Conflict at Walmart, Adam Reich and Peter Bearman

Judge Thy Neighbor: Denunciations in the Spanish Inquisition, Romanov Russia, and Nazi Germany, Patrick Bergemann

Concepts and Categories: Foundations for Sociological and Cultural Analysis, Michael T. Hannan, Gaël Le Mens, Greta Hsu, Balázs Kovács, Giacomo

Negro, László Pólos, Elizabeth Pontikes, and Amanda J. Sharkey 


\section{THE CORSAIRS \\ OF SAINT-MALO}

Network Organization

of a Merchant Elite

Under the Ancien Régime

HENNING HILLMANN

Columbia University Press

New York 


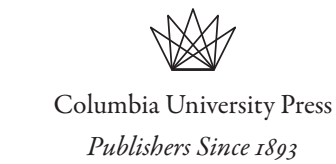

New York Chichester, West Sussex

cup.columbia.edu

Copyright (C) 2021 Columbia University Press

All rights reserved

Library of Congress Cataloging-in-Publication Data

Names: Hillmann, Henning, author.

Title: The corsairs of Saint-Malo : network organization of a merchant elite under the

Ancien Régime / Henning Hillmann.

Description: New York : Columbia University Press, [202I] | Includes bibliographical references and index.

Identifiers: LCCN 2020028804 (print) | LCCN 2020028805 (ebook) |

ISBN 9780231180382 (hardback) | ISBN 9780231180399 (trade paperback) |

ISBN 978023154266I (ebook)

Subjects: LCSH: Merchants-France-Saint-Malo. | Privateering-France-Saint-Malo.

| Harbors-France-Saint-Malo. | Saint-Malo (France)-Commerce. | France-Foreign economic relations. | France-Economic conditions-I7th century. | France-Economic conditions- 18 th century.

Classification: LCC HF $3560 . S_{3} \mathrm{H}_{55} 202 \mathrm{I}$ (print) | $\mathrm{LCC} \mathrm{HF}_{3560 . S_{3}}$ (ebook) |

DDC $_{3} 8 \mathrm{r} / .1094415-\mathrm{dc} 23$

LC record available at https://lccn.loc.gov/2020028804

LC ebook record available at https://lccn.loc.gov/2020028805

Columbia University Press books are printed on permanent and durable acid-free paper.

Printed in the United States of America

Cover design: Lisa Hamm

Cover image: Ambroise Louis Garneray, Capture of the prize ship Triton by the French corsair

Le Hasard. Archives départementales d'Ille-et-Vilaine, ${ }_{4} \mathrm{Fg} 63$.

Wikimedia Commons/Public domain. 
Enfermés le soir sous la même clef dans leur cité, les Malouins ne composaient qu'une famille.

-François-René de Chateaubriand, Mémoires d'Outre-Tombe (vol. I, book I, chap. 4) 
